\title{
Research on Remote Monitor and Safety About Substation Based on Wireless Private Network
}

\author{
Wei Lu, Xin Li \\ Shibei Power Supply Company, Shanghai Municipal Electric Power Company, Shanghai, China \\ Email address: \\ 13738674523@163.com (Wei Lu)
}

\section{To cite this article:}

Wei Lu, Xin Li. Research on Remote Monitor and Safety About Substation Based on Wireless Private Network. Automation, Control and Intelligent Systems. Vol. 3, No. 6, 2015, pp. 100-103. doi: 10.11648/j.acis.20150306.11

\begin{abstract}
With the technology of security video surveillance applied in unattended electric power substations, security management is the only way to modernize the power grid. The paper proposes a set of integrated solutions combining substation video, security and power supply based on the $4 \mathrm{G}$ Wireless Networks. It studies the remote video security system that integrates security and technical precautions with hardware and software combining. The system strengthens the safety and security management of unattended substation relying on the latest $4 \mathrm{G}$ private network technology and advanced hardware equipment.
\end{abstract}

Keywords: 4G Network, Remote Video Surveillance, Security and Technical Precautions, Solar Power Supply

\section{Introduction}

Nowadays, with the continuous development of China's electric power system, digital and automation is an inevitable trend of the development of electric power, unmanned substation and its safety management is the only way to modernize the power grid. With the usage of video surveillance system, the staff can inspect the operation [3]. It can be used to obtain the scene of the alarm information and review the accident through video recording.

Recently, the electrical power units in the substations are stolen and damaged throughout the country [1]. In order to ensure the safe operation of power facilities, reduce the loss of property, and ensure the normal operation of urban power network, it is necessary to take decisive measures [4]. High-tech means and effective management are useful methods to continuously reduce the occurrence of such events. At present, a large number of unmanned substations located in the jurisdiction of the Shibei Power Supply Company, Shanghai Municipal Electric Power Company [2]. The paper studies a remote visual security system that integrates security and technical precautions with hardware and software combination.

The paper proposes a set of integrated solutions combining substation video, security and power supply based on the $4 \mathrm{G}$ Wireless Networks. It studies the remote video security system that integrates security and technical precautions with hardware and software combining. It concluded that the system can realize the centralized management of substation, make important substation equipment, security monitoring points under real-time monitoring status and reduce the overall cost of operation and maintenance.

\section{Current Problems}

\subsection{Unable to Get the Running Status of the Devices in Time}

With a larger number of unmanned substation appears, the daily inspections and switching operations of the substation is still following the tradition of previous work mode because of various objective conditions, which lead to the relevant staff and the management must be present, particularly the substation in the remote areas, and it will cost a lot of manpower and resources on transportation.

\subsection{Complicated Construction of Video Surveillance System}

It is necessary to lay a great deal of wire for the video surveillance, and it is a difficult task. At the same time to build a monitoring system requires the laying of a large number of video lines, network lines, control lines and power lines, which has brought a huge cost of human and material resources. And ordinary video surveillance can only be viewed in special monitoring center, which cannot be viewed on mobile terminals or other devices, even worse it was subjected to the geographical limitations and networks in emergency situations. 


\subsection{Image Quality Await to Be Optimized}

Ordinary video camera image quality has been unable to meet the requirements of remote monitoring, the image quality will be damaged when the analog camera has been converted into digital, long distance transmission of analog signal, the signal attenuation is serious, the image quality is not good, the image resolution is fixed to PAL or NTSC, which cannot meet the requirements of higher resolution and higher resolution. Video signal dynamic range is small, it is difficult to meet specific application requirements, such as low noise, wide dynamic, high frame rates or other requirements, it also cannot achieve distribution, long distance transmission, sharing or other needs [5].

\subsection{Safety Hazards in Scenes}

Due to the wide distribution of substation; the environment surrounding is complex and unattended. The security issues of the substation is becoming more and more severer, the issue must be solved including fire prevention ${ }^{[4]}$, security, waterproof and so on, only in this way the safe operation of all the apparatus can be ensured.

\section{Research of Anti-theft Technology for Remote Monitoring}

\subsection{Integration of Remote Monitoring Platform and Security Equipment}

SDI HD infrared camera is used in the scene of video surveillance in substation, the shooting effect can up to $720 \mathrm{P}$, and the camera can be remote rotated 360 degree by the monitoring personnel for the camera using cloud control. Site security equipment (such as Infrared burglar, Smoke alarm, Vibration sensor) has been accessed to the host computer. The system uses the remote video terminal client platform to feedback the scene video, security alarm, mobile detection, automatic recording, it also supports remote viewing of PC terminal and mobile terminal equipment [6].

\subsection{Data Transmission Through 4G Network}

With the maturity of $4 \mathrm{G}$ network technology and the coverage of network, the China Telecom's $4 \mathrm{G}$ network can provide a more efficient, more stable video transmission network for the system. China Telecom's 4G network is still in trial operation stage, based on the virtual private network services that high-speed packet data telecommunications network had built for mobile customers, by which the corporate customers can get in touch with enterprise intranet Seamless and safely no matter where they are, at the same time the VPND network and the internet network can be isolated. The study based on the integrated of $4 \mathrm{G}$ network module and the site monitoring host computer, simultaneously, the $720 \mathrm{p}$ HD camera is used as the video capture unit, and transmission of $4 \mathrm{G}$ network ensured the high speed, safety, stability and reliability of the network data.

\subsection{Solar Backup Power Supply}

As the video surveillance and the security system need to maintain uninterrupted operation in 24 hours, in order to ensure that the system is also capable of running in emergency situations, as well as to ensure the storage security of the video hosting computer's data. All apparatus of the system will be used in two ways-powered, one way is mains combines UPS, and another way is solar with the spare battery, while the system utilize the dual power automatic transfer switch to realize the switching between the city electricity and the solar standby power supply, will not cause the malfunction, and meet for the requirement of high reliability. System can be in the field without the cable network and the external power supply [5], the normal operation to ensure that the full 24 hours monitoring.

\section{System Structure Introduction}

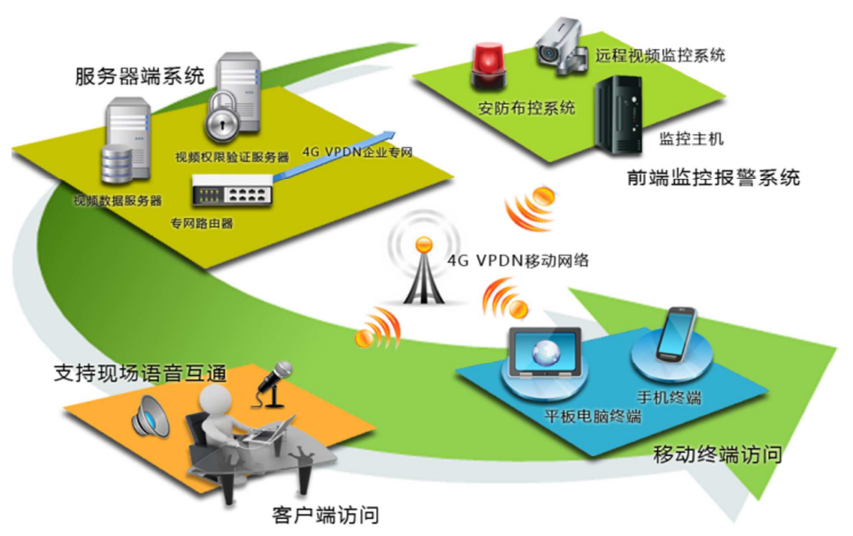

Fig. 1. Remote Monitoring System Configuration.

\subsection{Entire Structure Design}

Remote anti-theft monitoring system composes of the monitored alarm system, the power supply system and the Server-side system, and the monitored alarm system is contributed by remote monitoring system and site security surveillance system.

Hardware devices of remote monitoring system are composed of high-definition network camera and remote monitor $4 \mathrm{G}$ computers.

The devices of site security surveillance system are composed of anti-theft monitoring computer, infrared alarm, smoke alarm, sound and light alarm, vibration alarm, alarm siren, noise reduction devices and so on.

The power supply system composes of UPS host, dual power switch, inverter, solar photovoltaic panels, and battery pack and so on.

The server composes of remote video surveillance server, Video data server, Dedicated 4G VPDN network, VPDN router.

The system transmissions the remote video images and site security surveillance system data to server system through dedicated $4 \mathrm{G}$ network by alarm system. Users connect to the 
server system through a client remote system and can check live video images and alarm information feedback. At the same time, the system supports table PCs and mobile phones monitoring on site.

\subsection{System Feature}

The system has a reasonable structure design and advanced technology concepts. It's based on live video surveillance and to assist in security anti-theft monitoring. The system using the advanced video compression data, security monitoring and video monitoring combine with $4 \mathrm{G}$ network perfectly, meanwhile, the system supports two monitoring modes of $\mathrm{C} / \mathrm{S}$ and $\mathrm{B} / \mathrm{S}[7]$.

The design of this system is based on the international protocol standard. All devices can be included in the system as long as they meet the agreement.

Integrated platform has a powerful management function. System combines video surveillance, integrated platform alarm, message alarm in one, achieving an application platform to control all devices in the management system. The system is based on security to assist power production, unified platform for centralized management, real-time monitoring of all front-end equipment and reduce user maintenance costs.

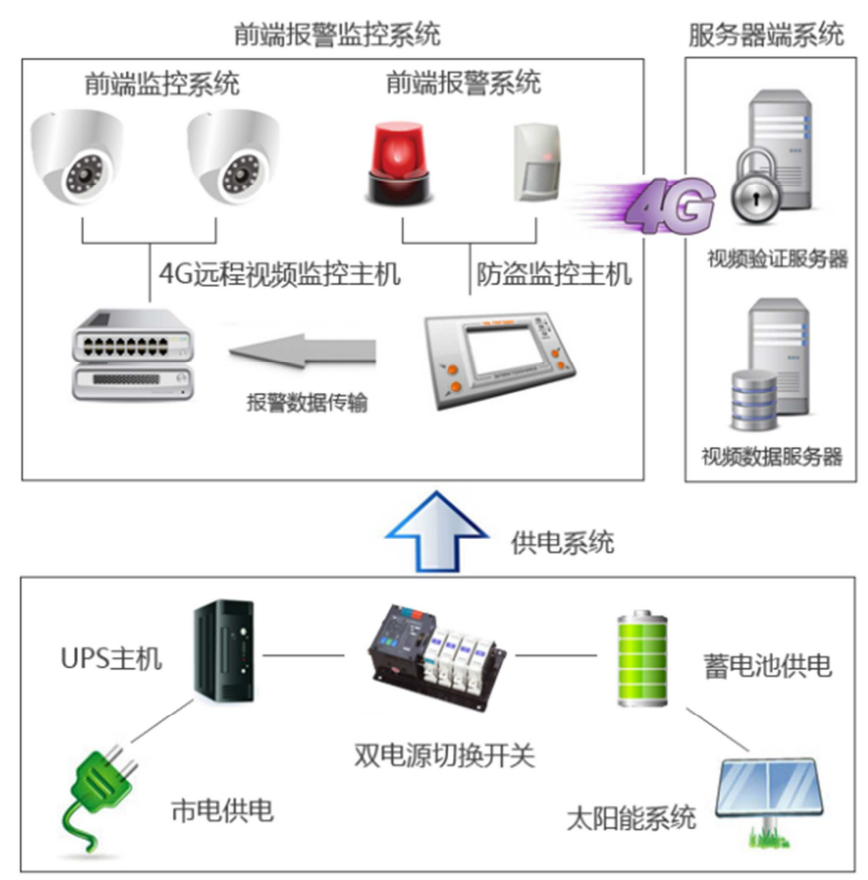

Fig. 2. Configuration of the Safety and Power Supply System.

\section{Software Features}

\subsection{Data Flow Control of the Wireless Network}

By using 4G mobile data transmission network, we need to solve the problem of large transport stream, otherwise it will lead to uncontrolled operation and maintenance costs. Research will use the local video store, online video viewing, and emergency data upload program to reduce the transmission of video surveillance traffic. Local video store, online video viewing:

Traditional video surveillance, the live video monitoring continues to transmit data to a remote server, the server-side data storage, you can connect to the server view back when you need to see the video. So there will be a lot of video data transmission between server and monitoring, resulting the waste of network resource and the data redundant of server [8].

Research will store all monitoring data directly in the local monitoring host disk space, all data is exchanged with other server when monitoring, and server-side unified manages the on-site monitoring equipment. When users need to watch back, using a client platform to connect system server-side. System allocates monitoring disk space through server-side, at the same time data traffic will be produced and the traffic transmission will be reduced.

\subsection{Data Save in Incident}

If something emergency happened in transformer substation, the system will start the motion detection function. If non-normal mobile phenomenon happened in some specific areas, the system will automatically start recording function. System will select automatically recording function when alarm of system integration arm, it will automatically start recording function when the alarm Detectors (such as infrared alarm) alarm occurs. The recording time can be set in advance; the days of video file can be adjusted.

In this case the video data will be automatically uploaded to a remote server's hard disk through network and notify the administrator unexpected things has happened, so administrator has access to the site video view and it can provide the evidence for events [9].

\subsection{Remote Patrol}

With the constant improvement of communication network systems and the increasing unmanned substations, although the power sector has real-time monitoring data on the grid, they cannot grasp the site operating conditions and check field devices image. Using remote video inspection technology, we will enable access to the image information within the substation and a variety of alarm information, sector can responses and records the operation of substation equipment running key parts more quickly, intuitively, accurately [10].

Administrators can connect a video camera inside the substation configuration, and understand the station in real time through the client platform.

The resolution of station configuration multiple SDI HD cameras can reach to 720P.That can achieve visualization monitoring and scheduling of substation to ensure unmanned substations' running become more safe and reliable with the help of data transmission of $4 \mathrm{G}$ high-speed network.

\subsection{Record Video}

The system can be set multiple periods of multiple front-end monitoring equipment for recording. Data will be stored in the hard disk of video surveillance system server or front-end devices. Timer mode can be selected as single 
recording or scheduled recording and each time can be set separate, recording speed can be adjusted.

\subsection{Map Showing Substation Location}

The system can be positioned by the GPS of front-end monitoring equipment host. It's useful for this system to unified management and visual show of all devices by using GPS technology to combine substation with electronic maps. It's shown in figure.

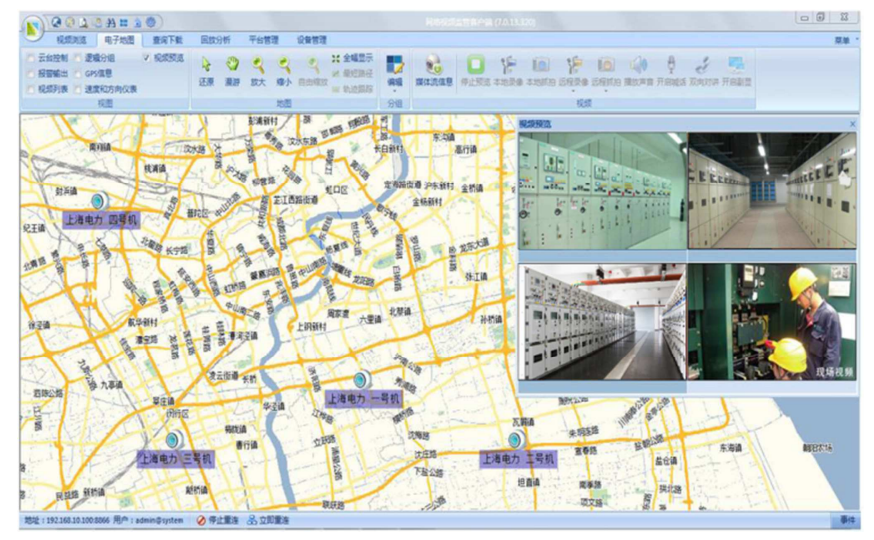

Fig. 3. Electronic Map of Substation.

\section{Conclusion}

Achieving substation automation and modernization of management, continuously improving the safety and reliability of the substation operation and maintenance, improving management efficiency for the planning and construction of video monitoring system and the complete security, anti-technology within the existing substation are the main goals of this article.

Optimizing equipment power supply system by using high-speed secure wireless network technology and integrated substation video surveillance and security equipment. The system can realize the centralized management of substation, make important substation equipment, security monitoring points under real-time monitoring status and reduce the overall cost of operation and maintenance.

\section{References}

[1] LIU qingli, LIU jingbo, DENG ying. Video analysis technology in unmanned substations Monitoring. Shanxi Electric Power, 2010, pp.73-76.

[2] LUO lei, ZANG shaohua. Substation remote inspection system wireless communication technology. Power and Technology Science, 2014, pp.92-96.

[3] DING shuwen, WANG cheng. Key Technical Problems Intelligent Substation. For electricity, 2012, pp.9-12+47.

[4] LIU qin, LIU jian. Implementation and Application of unmanned substation Remote Monitoring System Analysis. China Electric Power Education, 2012, pp. 141-142.

[5] LU shen. Remote monitoring system in unmanned substations. Huzhou Teachers College, 2013, pp. 32-35.

[6] SUN jingming. Substation network remote monitoring system application. China Science and Technology Information, 2014, pp.122-124.

[7] PAN yueqing, LUO jingfeng, Monitoring technology in power theft Work. Wireless Internet Technology, 2012, pp.2-7.

[8] MIAO wennan, Car Alarm Monitoring System. 2014, pp.22-24.

[9] ZEN peng, YU haibing, Industrial Wireless Network WIA standard system and technologies. Automation Panorama, 2012, pp.24-27.

[10] XU haiyan, LIU jingwei, The fourth generation of mobile communication technology research. Wireness Internet Technology, 2015, pp.20-21. 